\author{
F. W. Williams \\ D. Kennedy \\ Cardiff School of Engineering \\ University of Wales Cardiff \\ P.O. Box 917 \\ Cardiff CF2 1XH, U.K.
}

\title{
Review
}

\section{Accelerated Solutions for Transcendental Stiffness Matrix Eigenproblems}

\begin{abstract}
This article outlines many existing and forthcoming methods that can be used alone, or in various combinations, to accelerate the solutions of the transcendental stiffness matrix eigenproblems that arise when the stiffness matrix is assembled from exact member stiffnesses, which are obtained by solving the member differential equations exactly. Thus distributed member mass and/or the flexural effect of axial loading are incorporated exactly, and the solutions are the natural frequencies for vibration problems or the critical load factors for buckling problems. (C) 1996 John Wiley \& Sons, Inc.
\end{abstract}

\section{INTRODUCTION}

When member stiffness matrices are obtained by solving the differential equations, which include the distributed member mass and/or the destabilizing effect of axial force, their elements are transcendental functions of frequency and/or load factor. Hence the overall stiffness matrix $\mathbf{K}$ of a structure assembled from such members is a transcendental function of the eigenparameter, $\rho$, which is the frequency in vibration problems or the load factor in buckling problems. Therefore, solving the transcendental eigenproblem,

$$
\mathbf{K} \mathbf{D}=\mathbf{0},
$$

gives the eigenvalues as the natural frequencies of (optionally) loaded structures for vibration problems, or the critical load factors of the live load system for buckling of structures that also (optionally) carry dead load.

The earliest solutions, such as that of Simpson and Tabarrok (1968), plotted $|\mathbf{K}|$ versus $\rho$ and hence the values of $\rho$ at which $|\mathbf{K}|=0$ (i.e., at which the curve crossed or touched the $\rho$ axis) were the eigenvalues. Because the curves can be very badly behaved, $|\mathbf{K}|$ had to be calculated at very fine intervals of $\rho$, i.e., many iterations were required, and even then eigenvalues could be missed. For example all eigenvalues for which the displacement vector $\mathbf{D}$ is nodal in the mode, i.e., $\mathbf{D}=\mathbf{0}$ modes, were inevitably missed. The Wittrick-Williams algorithm (Williams and Wittrick, 1970; Wittrick and Williams, 1973) had the principal advantage of ensuring that eigenvalues could never be missed by calculating $J$, the number of eigenvalues between zero and the current trial value $\rho_{\mathrm{t}}$ of the eigenparameter, from

$$
J=J_{0}+s\{\mathbf{K}\} .
$$

Here $J_{0}$ is the value that $J$ would have if $\mathbf{D}$ were clamped, i.e., $\mathbf{D}=\mathbf{0}$, and $s\{\mathbf{K}\}$ is calculated as the number of negative leading diagonal elements of $\mathbf{K}^{\Delta}$, the upper triangular matrix obtained from $\mathbf{K}$ by the usual form of Gauss elimination. Thus the rows of $\mathbf{K}$ are taken as pivotal in order and multiples of the pivotal row are added to unscaled 
succeeding rows, so that $|\mathbf{K}|$ is readily computed as the product of the leading diagonal elements of $\mathbf{K}^{\Delta}$.

Coincident eigenvalues were automatically detected, their multiplicity being the difference between the values of $J$ computed at minutely separated upper and lower bound $\rho$ values. Hopper and Williams (1977) found that except for $\mathbf{D}=\mathbf{0}$ modes, replacing the null right-hand side of Eq. (1) by a random force vector and then solving with $\mathbf{K}$ computed with $\rho_{\mathrm{t}}$ close to the eigenvalue gave very good modes. This also worked for coincident eigenvalues, by using a number of random right-hand sides equal to the multiplicity of the eigenvalue.

A secondary, but important, advantage of the Wittrick-Williams algorithm is that because eigenvalues cannot be missed, it gives much faster solutions because it is no longer necessary to perform iterations at fine intervals of $\rho$. For example, for many years the bisection routines of Williams and Wittrick (1970) were favored and these greatly reduced the number of iterations needed.

\section{WELL-ESTABLISHED ACCELERATION METHODS}

Wittrick and Williams (1970) realized from the outset that their algorithm could still be applied if the order of the $\mathbf{K}$ of Eqs. (1) and (2) is reduced to any desired extent without approximation, even to the limit of making $\mathbf{K}$ a scalar, by the exact form of substructuring used in static analysis. Thus $\mathbf{D}$ is ordered to have internal substructure displacements first, and then Gauss elimination is arrested after all rows of $\mathbf{K}$ corresponding to these internal displacements have been eliminated. Williams (1973a) showed that this often gives faster eigensolutions than sparse matrix methods do, because identical substructures need only be analyzed once. For practical prismatic stiffened panels analyzed by exact strip theory, Williams (1972) showed that one level of substructuring saved about $67 \%$ of the solution time otherwise needed, rising to $80 \%$ when multilevel substructuring was used. Previously, Williams (1971) obtained even larger savings for general structures containing portions with many identical repetitions, by recursively doubling the substructure size by connecting two of the previous substructures together, starting with two of the identical repetitions.
It was realized that Eq. (2) could be used to design structures without the need to analyze them first. Thus Williams (1973b) modified structures intuitively, to move inconveniently situated eigenvalues or to create a frequency band free of natural frequencies, by calculating $J$ at each end of the band for each trial design in turn, until a trial design was found for which these two values of $J$ were equal. Alternatively, Williams and Wright (1978) factored all the thicknesses of a prismatic plate assembly to make it just stable at a required buckling load. Such procedures greatly accelerate solution times by avoiding the need to fully analyze each trial design and have been used to advantage by Butler and Williams (1992) in the optimum design option of the recent prismatic plate assembly analysis or design software VICONOPT, which is now well established in the aerospace industry.

Williams and Anderson (1983) used Lagrangian multipliers to connect structures together by coupling their transcendental stiffness matrices, and to couple the transcendental stiffness matrices of appropriate wavelengths of response of prismatic structures to give an approximate match to awkward support conditions, etc. This approach yields a constrained matrix with the transcendental stiffness matrices on its diagonal. In the latter case, Anderson et al. (1983) observed that calculation of the eigenvalues can be very considerably accelerated because the wavelengths can only couple in certain combinations. A major further acceleration was achieved by Williams and Anderson (1985) for transversely repetitive structures, by analyzing a single repeating portion alone, hence greatly reducing the order of $\mathbf{K}$ in Eq. (2) at the expense of having to repeat the analysis for several assumed wavelengths of transverse response.

It is also possible to analyze rotationally periodic structures by analyzing a single repeating portion for each possible circumferential wavelength in turn. This too accelerates convergence on the exact eigenvalues very considerably, particularly if complex arithmetic is used, with the special procedures of Williams (1986a) used to permit nodes and members to lie along the axis of rotational periodicity. Such procedures were extended by Williams (1986b) to permit rotational periodicity of substructures to be used even when the parent structure is not periodic, so long as the only connections are at the two ends of the axis of rotational periodicity of each substructure. This is the case in the earlier work of Williams 
and Howson (1978) and Banerjee and Williams (1984) where the substructure is a collapsible stayed column suitable for use in space. Further extensions by Wu et al. (1996) permit rotationally periodic substructures to be connected in any required way and at a number of connection nodes to an arbitrary parent structure. Williams et al. (1993) also recently extended these procedures, using the Lagrangian multiplier approach, to enable exact eigenvalues to be obtained by analyzing only one repeating portion of a rotationally periodic structure, e.g., a dome, for which the support system repeats round the circumference much less frequently than the structure does, so that the portion analyzed is much smaller than the support spacing.

Williams and Kennedy (1988b) accelerated convergence by replacing bisection, when the curve is suitably shaped, by carefully designed routines for following the curve of $|\mathbf{K}|$ versus $\rho$. Such routines switch to bisection when the curve departs in major ways from being parabolic, has poles in the range considered, etc., while ensuring convergence by using the Wittrick-Williams algorithm at each iteration. Such curve following is approximately twice as fast as bisection and appears to be quicker than the attractive earlier Newtonian method of Simpson (1984). Kennedy and Williams (1991) even used it to find coincident eigenvalues by plotting the $n$th root of $|\mathbf{K}|$, where $n$ is the number of eigenvalues potentially coincident, calculated as the difference between the values of $J$ at the current upper and lower bounds on the eigenvalues. Convergence can also be accelerated by using methods that improve on the speed of the Gauss elimination used to reduce $\mathbf{K}$ to $\mathbf{K}^{\Delta}$, e.g., the Gauss-Doolittle method of Williams and Kennedy (1988a), which typically saves up to $25 \%$ of the elimination time.

\section{RECENT AND FUTURE WORK}

Hopper et al. (1980) showed that using the values of $\mathbf{K}$ at two widely separated values, $\rho_{1}$ and $\rho_{2}$, of $\rho$ to linearize the eigenproblem to become

$$
(\mathbf{A}-\rho \mathbf{B}) \mathbf{D}=\mathbf{0},
$$

where $\mathbf{A}$ and $\mathbf{B}$ are calculated from

$$
\left.\begin{array}{l}
\mathbf{A}-\rho_{1} \mathbf{B}=\mathbf{K}\left(\rho_{1}\right) \\
\mathbf{A}-\rho_{2} \mathbf{B}=\mathbf{K}\left(\rho_{2}\right)
\end{array}\right\}
$$

gave lower bounds on the exact eigenvalues lying between $\rho_{1}$ and $\rho_{2}$ and upper bounds otherwise. Ye and Williams (1994) recently proved that this result is also true for the constrained matrices described above in connection with the application of Lagrangian multipliers, so that $\mathbf{K}$ is now the contrained matrix when applying Eq. (4). This led to a similar bounding result for the quadratic eigenvalue problem that exactly represents the constrained transcendental problem at three widely separated values of $\rho$, namely at $\rho_{1}, \rho_{2}$, and $\rho_{3}$. Hence the eigenvalue problem becomes

$$
\left(\mathbf{A}-\rho \mathbf{B}-\rho^{2} \mathbf{C}\right) \mathbf{D}=\mathbf{0},
$$

where $\mathbf{A}, \mathbf{B}$, and $\mathbf{C}$ can be calculated from

$$
\left.\begin{array}{l}
\mathbf{A}-\rho_{1} \mathbf{B}-\rho_{1}^{2} \mathbf{C}=\mathbf{K}\left(\rho_{1}\right) \\
\mathbf{A}-\rho_{2} \mathbf{B}-\rho_{2}^{2} \mathbf{C}=\mathbf{K}\left(\rho_{2}\right) \\
\mathbf{A}-\rho_{3} \mathbf{B}-\rho_{3}^{2} \mathbf{C}=\mathbf{K}\left(\rho_{3}\right)
\end{array}\right\} \quad\left(\rho_{1}<\rho_{2}<\rho_{3}\right)
$$

Ye and Williams (1995b) showed that, subject to certain conditions, this approximation gives lower bounds on exact eigenvalues lying below $\rho_{1}$ or between $\rho_{2}$ and $\rho_{3}$, but otherwise gives upper bounds. Convergence on the exact eigenvalues can be achieved, to any desired accuracy, by either of the linear or quadratic approximations described above, by making $\rho_{1}$ and $\rho_{2}$ (and $\rho_{3}$ in the quadratic case) progressively closer together and choosing their positions to ultimately obtain close upper and lower bounds on the required eigenvalue(s). Ye and Williams (1995a) recently showed that such a convergence procedure considerably accelerates convergence on the fundamental eigenvalue of prismatic plate assemblies, e.g., saving about $30 \%$ of the solution time. An attractive Lanczos-based technique was also recently developed by Smith et al. (1993) and gave very substantial time savings for large matrices $\mathbf{K}$ when compared with the curve following method of Williams and Kennedy (1988b), when substructuring was not used, although recent correspondence by Kennedy and Williams (1994) qualifies the extent of these claimed time savings.

Recent work by Powell et al. (1996) achieves time savings of $50-75 \%$ by including Lagrangian multipliers within substructures. Ronagh et al. (1995) recently found $\mathbf{D}=\mathbf{0}$ modes without altering $\mathbf{K}$ or $\mathbf{K}^{\Delta}$, by proceeding with the mode finding as if substructures had been used to represent the 
members. Hence, in effect, this incorporates their internal freedoms within $\mathbf{D}$, so that $\mathbf{D} \neq \mathbf{0}$ and therefore the mode can be found by the random force vector method.

The bisection and curve following procedures described in the previous section offer great scope for the application of parallel computing. Good efficiency (e.g., a speedup of 6.57 when using eight processors, i.e., an efficiency of approximately $82 \%$ ) was recently demonstrated by Watkins et al. (1996b), when simultaneously finding a large number of natural frequencies of a frame, simply by allocating different eigenvalues to different processors and reallocating them if processors become idle. Recently Watkins et al. (1996a) showed that the advantages of the earlier rapid Gauss-Doolittle form of reduction of $\mathbf{K}$ to $\mathbf{K}^{\Delta}$ (Williams and Kennedy, 1988a) are retained when parallel computing is employed, although there is a marked deterioration in solution times when more than a few processors are used due to the volume of interprocessor communication. The hybrid parallelization of Kennedy et al. (1995), in which several of the available processors are allocated to each eigenvalue, with the processors allocated to any given eigenvalue cooperating in parallel Gauss-Doolittle reduction, achieved time savings of about $20 \%$ over the method of Watkins et al. (1996b). Watkins et al. (1996c) showed that the performance of such methods on distributed memory parallel computers depends crucially on the ratio of calculation speed to interprocessor communication speed, so that machine-independent measures of performance can be obtained by simulation. Future work includes a parallel solution of the linearlized eigenproblem of Eq. (3) and the analysis of substructures in parallel.

\section{CONCLUSIONS}

The use of the Wittrick-Williams algorithm gives substantial time savings even when used with simple bisection routines, because it is no longer necessary to calculate $|\mathbf{K}|$ at fine intervals of the eigenparameter. Such solution times can be further halved by curve following methods, reduced by perhaps $70 \%$ more by substructuring, reduced much further still if the structure is linearly or rotationally periodic (which enables several computations with the very small matrix of the repetitive portion to replace computation with the matrix of the full structure), and can still be further reduced by introducing linear or quadratic matrix pencil approximations to the eigenproblem at the earliest permissible stage of convergence. When Lagrangian multipliers are used (e.g., to couple responses to achieve desired boundary conditions), still further acceleration of the convergence is achievable because the wavelengths can only couple in certain combinations. This enables the efficient analysis of prismatic plate assemblies and repetitive space frames for which the supports repeat less frequently than the structure.

All of the computations are extremely well adapted to the efficient use of parallel computation, and design can be performed without the need to fully analyze all intermediate design stages. Coincident eigenvalues can be computed extremely efficiently. The associated mode shapes can be found very efficiently, even when all the degrees of freedom of the system that correspond to $\mathbf{K}$ are null in the mode.

\section{REFERENCES}

Anderson, M. S., Williams, F. W., and Wright, C. J., 1983, "Buckling and Vibration of Any Prismatic Assembly of Shear and Compression Loaded Anisotropic Plates with an Arbitrary Supporting Structure," International Journal of Mechanical Sciences, Vol. 25, pp. 585-596.

Banerjee, J. R., and Williams, F. W., 1984, "Evaluation of Efficiently Computed Exact Vibration Characteristics of Space Platforms Assembled from Stayed Columns," Journal of Sound and Vibration, Vol. 95, pp. 405-414.

Butler, R., and Williams, F. W., 1992, “Optimum Design Using VICONOPT, a Buckling and Strength Constraint Program for Prismatic Assemblies of Anisotropic Plates," Computers and Structures, Vol. 43, pp. 699-708.

Hopper, C. T., Simpson, A., and Williams, F. W., 1980, "A Study of the Bounds on Eigenvalues of a Transcendental Dynamic Stiffness Matrix Provided by a Simply Derived Linear Matrix Pencil,' Journal of Structural Mechanics, Vol. 8, pp. 365-422.

Hopper, C. T., and Williams, F. W., 1977, "Mode Finding in Nonlinear Structural Eigenvalue Calculations," Journal of Structural Mechanics, Vol. 5, pp. 255-278.

Kennedy, D., Watkins, W. J., and Williams, F. W., 1995, "Hybrid Parallel Computation of Transcendental Structural Eigenvalues," AIAA Journal, Vol. 33, pp. 2194-2198.

Kennedy, D., and Williams, F. W., 1991, “More Efficient Use of Determinants to Solve Transcendental 
Structural Eigenvalue Problems Reliably," Computers and Structures, Vol. 41, pp. 973-979.

Kennedy, D., and Williams, F. W., 1994, “Discussion of 'A Lanczos-Based Technique for Exact Vibration Analysis of Skeletal Structures',' International Journal for Numerical Methods in Engineering, Vol. 37, pp. 3197-3198.

Powell, S. M., Kennedy, D., and Williams, F. W., 1996, "Efficient Multi-Level Substructuring with Constraints for Buckling and Vibration Analysis of Prismatic Plate Assemblies," to appear.

Ronagh, H. R., Lawther, R., and Williams, F. W., 1995, "Calculation of Eigenvectors with Uniform Accuracy," Proceedings of ASCE, Journal of Engineering Mechanics, Vol. 121, pp. 948-955.

Simpson, A., 1984, "On the Solution of $\mathbf{S}(\omega) \mathbf{x}=\mathbf{0}$ by a Newtonian Procedure,' Journal of Sound and Vibration, Vol. 97, pp. 153-164.

Simpson, A., and Tabarrok, B., 1968, "On Kron's Eigenvalue Procedure and Related Methods of Frequency Analysis,"' Quarterly Journal of Mechanics and Applied Mathematics, Vol. 21, pp. 1-39.

Smith, H. A., Sorensen, D. C., and Singh, R. K., 1993, "A Lanczos-based Technique for Exact Vibration Analysis of Skeletal Structures," International Journal for Numerical Methods in Engineering, Vol. 36, pp. 1987-2000.

Watkins, W. J., Kennedy, D., and Williams, F. W., 1996a, "Efficient Parallel Gauss-Doolittle Matrix Triangulation," to appear.

Watkins, W. J., Kennedy, D., and Williams, F. W., 1996b, "Efficient Parallel Solution of Structural Eigenvalue Problems,' Advances in Engineering Software Incorporating Computing Systems in Engineering, to appear.

Watkins, W. J., Kennedy, D., and Williams, F. W., 1996c, "On Estimating Machine Dependency of Fine and Coarse Grain Parallel Structural Computations,"' Microcomputers in Civil Engineering, to appear.

Williams, F. W., 1971, "Natural Frequencies of Repetitive Structures," Quarterly Journal of Mechanics and Applied Mathematics, Vol. 24, pp. 285-310.

Williams, F. W., 1972, "Computation of Natural Frequencies and Initial Buckling Stresses of Prismatic Plate Assemblies," Journal of Sound and Vibration, Vol. 21, pp. 87-106

Williams, F. W., 1973a, “Comparison Between Sparse Stiffness Matrix and Sub-Structure Methods," International Journal for Numerical Methods in Engineering, Vol. 5, pp. 383-394.

Williams, F. W., 1973b, "Rapid Analysis of the Effects of Structural Modifications on Inconveniently Situated Eigenvalues," Computers \& Structures, Vol. 3, pp. 1465-1471.

Williams, F. W., 1986a, “An Algorithm for Exact Eigenvalue Calculations for Rotationally Periodic Structures," International Journal for Numerical Methods in Engineering, Vol. 23, pp. 609-622.

Williams, F. W., 1986b, "Exact Eigenvalue Calcula- tions for Structures with Rotationally Periodic Substructures," International Journal for Numerical Methods in Engineering, Vol. 23, pp. 695-706.

Williams, F. W., and Anderson, M. S., 1983, "Incorporation of Lagrangian Multipliers into an Algorithm for Finding Exact Natural Frequencies or Critical Buckling Loads,' International Journal of Mechanical Sciences, Vol. 25, pp. 579-584.

Williams, F. W., and Anderson, M. S., 1985, "Buckling and Vibration Analysis of Shear-Loaded Prismatic Plate Assemblies with Supporting Structures, Utilizing Symmetric or Repetitive Cross-Sections, ' in Aspects of the Analysis of Plate Structures-A Volume in Honour of W. H. Wittrick, edited by D. J. Dawe, R. W. Horsington, A. G. Kamtekar, and G. H. Little, pp. 51-71. Oxford University Press, Oxford, U.K.

Williams, F. W., and Howson, W. P., 1978, "Concise Buckling, Vibration and Static Analysis of Structures Which Include Stayed Columns," International Journal of Mechanical Sciences, Vol. 20, pp. 513-520.

Williams, F. W., and Kennedy, D., 1988a, "Fast Gauss-Doolittle Matrix Triangulation," Computers \& Structures, Vol. 28, pp. 143-148.

Williams, F. W., and Kennedy, D., 1988b, "Reliable Use of Determinants to Solve Non-Linear Structural Eigenvalue Problems Efficiently," International Journal for Numerical Methods in Engineering, Vol. 26, pp. 1825-1841.

Williams, F. W., Kennedy, D., Song, L. M., and Jemah, A. K., 1993, "Natural Frequencies of Rotationally or Linearly Repetitive Space Frames," in Space Structures 4, Proceedings of the 4th International Conference on Space Structures, edited by G. A. R. Parke, and C. M. Howard, pp. 423-431. Vol. 1, Thomas Telford, London.

Williams, F. W., and Wittrick, W. H., 1970, "'An Automatic Computational Procedure for Calculating Natural Frequencies of Skeletal Structures,"' International Journal of Mechanical Sciences, Vol. 12, pp. 781-791.

Williams, F. W., and Wright, C. J., 1978, “A DesignOrientated Alternative to Finding the Initial Buckling Loads of Prismatic Plate Assemblies," The Aeronautical Journal of the Royal Aeronautical Society, Vol. 82, pp. 399-402.

Wittrick, W. H., and Williams, F. W., 1973, “'An Algorithm for Computing Critical Buckling Loads of Elastic Structures," Journal of Structural Mechanics, Vol. 1, pp. 497-518.

Wu, G. F., Williams, F. W., and Kennedy, D., 1996, "Exact Eigenvalue Calculations for Partially Rotationally Periodic Structures," Computers \& Structures, to appear.

Ye, J. Q., and Williams, F. W., 1994, "Bounding Properties for Eigenvalues of a Transcendental Constrained Matrix by Using a Simple Matrix Pencil," Journal of Sound and Vibration, Vol. 177, pp. 282-287. 
Ye J. Q., and Williams, F. W., 1995a, “A Successive Bounding Method to Find the Exact Eigenvalues of Transcendental Stiffness Matrix Formulations," International Journal for Numerical Methods in Engineering, Vol. 38, pp. 1057-1067.
Ye J. Q., and Williams, F. W., 1995b, “Bounding Properties for Eigenvalues of a Transcendental Dynamic Stiffness Matrix by Using a Quadratic Matrix Pencil," Journal of Sound and Vibration, Vol. 184, pp. 173-183. 

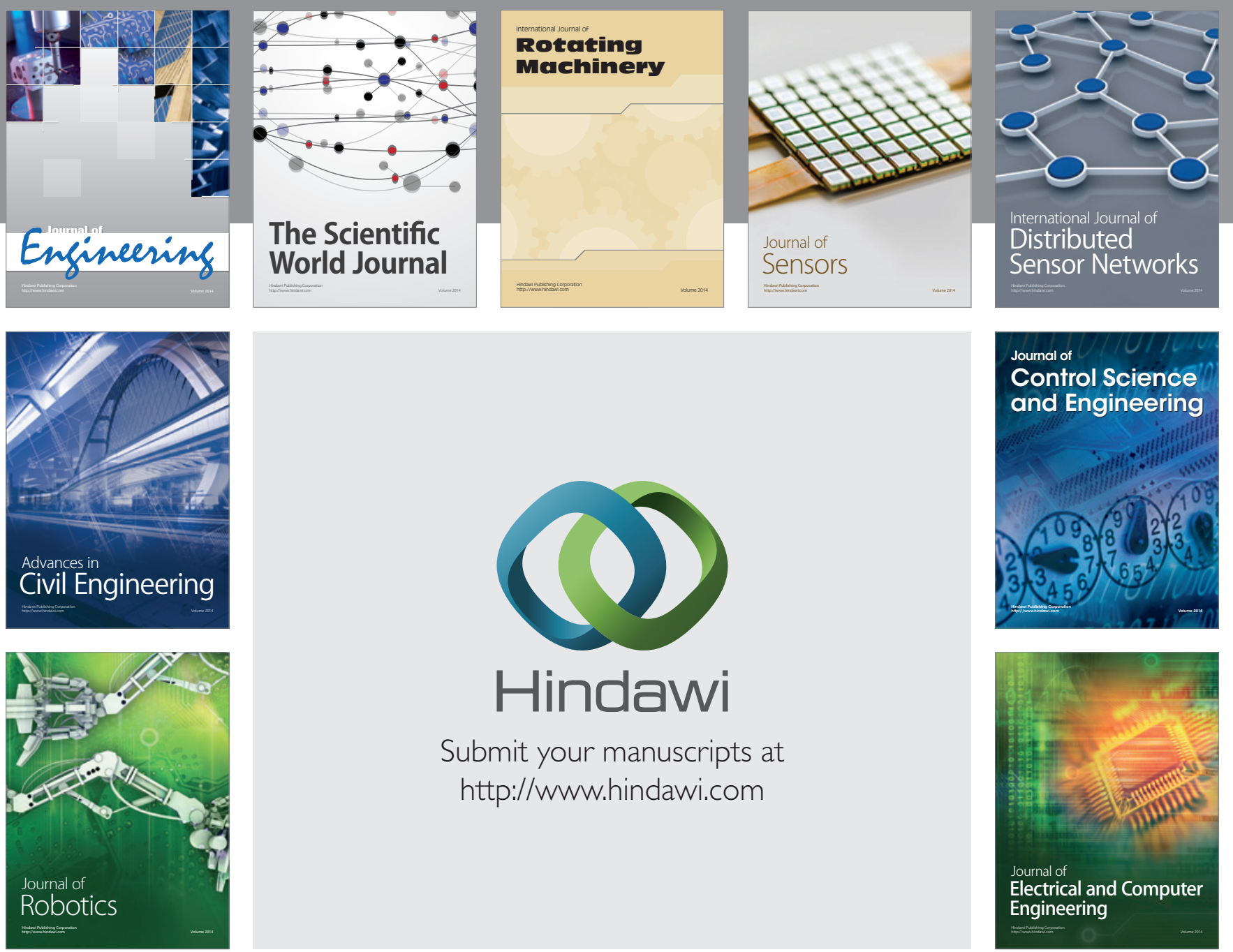

Submit your manuscripts at

http://www.hindawi.com
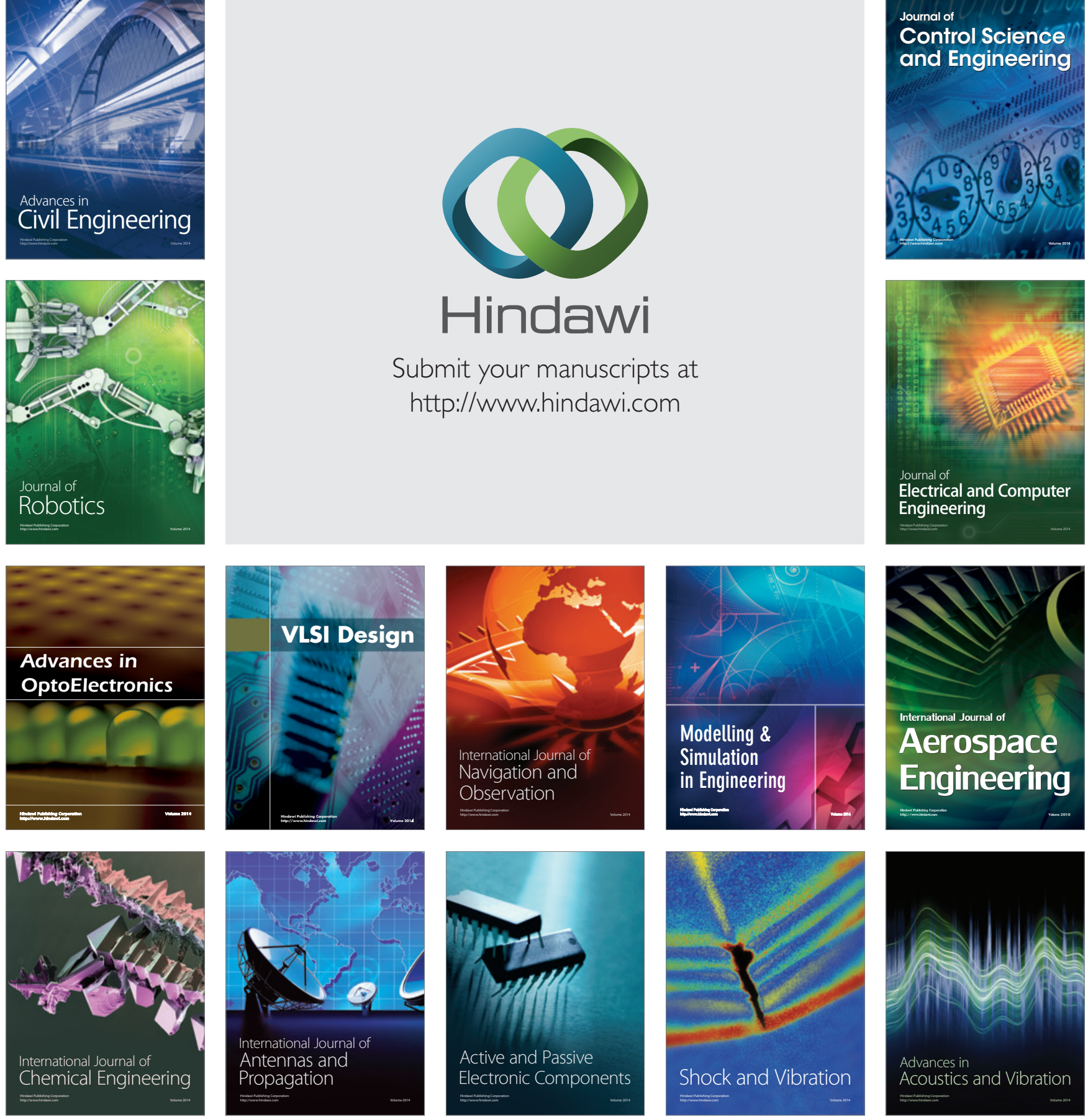